\title{
Actualización de los criterios STOPP-START: una herramienta para la detección de medicación potencialmente inadecuada en ancianos
}

New version of STOPP-START criteria: Tools for the detection of potentially inappropriate medications in the elderly

Cristian Galloł, Javier Vilosioł y J.Saimovicił

\begin{abstract}
Resumen
La utilización inapropiada de medicamentos en los adultos mayores conlleva frecuentemente la génesis de reacciones adversas debido a los cambios fisiológicos asociados a la edad. Esto aumenta la morbimortalidad determinando un mayor número de admisiones hospitalarias, con incremento de la utilización de recursos sanitarios y gastos en salud. Para poder optimizar la prescripción de medicamentos en los ancianos se han desarrollado en los últimos años herramientas implícitas y explicitas. Los criterios explícitos STOPP-START publicados en el 2008 se han impuesto como referencia en Europa aplicándose en diferentes ámbitos asistenciales. En este artículo se presenta una actualización de estos criterios realizada en el año 2014
\end{abstract}

\section{Abstract}

Improper use of drugs in older adults more often leads to adverse drug reactions due to the physiological changes associated with aging. This situation increases morbidity and mortality and determinates more hospital admissions, more use of health resources and health expenditures. In order to optimize the drug prescriptions in elderly, implicit and explicit tools have been developed in recent years. The STOPP-START 2008 explicit criteria have been imposed as a reference in Europe and have been applied in several areas of assistance. This paper presents an update of these criteria, accomplished in 2014.

Gallo C, Vilosio J. Actualización de los criterios STOPP-START, una herramienta para la detección de medicación potencialmente inadecuada en ancianos. Evid Act Pract Ambul 2015;18(4):124-129. Oct-Dic.

\section{El Problema}

Los adultos mayores reciben un número aumentado de medicamentos en comparación con los más jóvenes ${ }^{1}$, estando más expuestos a las reacciones adversas a medicamentos (RAM), definidas por la organización mundial de la salud (OMS) como cualquier respuesta nociva o indeseable a un fármaco que ocurre a las dosis usualmente utilizadas para profilaxis, diagnóstico y tratamiento de una patología.

Esta asociación se debe a los cambios fisiológicos asociados a la edad, el diferente comportamiento farmacocinético y farmacodinámico, la presencia de comorbilidades, malnutrición y caquexia, la polifarmacia (uso simultáneo de más de cuatro fármacos), la atención por múltiples especialistas y en diferentes niveles asistenciales, y a algunas características de la interacción con el paciente en el momento de la prescripción ${ }^{2,3}$ motivos que hacen que se excluya a la población de edad avanzada de la mayoría de los estudios sobre eficacia y seguridad de los medicamentos ${ }^{4,5}$.

La utilización inapropiada de los medicamentos plantea consecuencias sanitarias que se expresan a través de aumento en la morbimortalidad y consumo de recursos sanitarios, por lo que es considerado un problema de salud pública ${ }^{6}$.

En los Estados Unidos, los adultos mayores consumen el 32\% de todos los medicamentos recetados, y en ellos se ha reportado una prevalencia de RAM del $35 \%^{7}$, las cuales a su vez se estima que son responsables de hasta un $30 \%$ de los ingresos hospitalarios en esta población ${ }^{8,9}$

En Argentina, hay escasos datos sobre el consumo de medicamentos en ancianos, probablemente por la dificultad que supone el tipo de contratos que los principales financiadores establecen con la industria farmacéutica y/o proveedores. Un estudio descriptivo realizado en 2009 en la Provincia de Buenos Aires sobre 370 pacientes institucionalizados, mostró que el $27 \%$ de los adultos consume más de cinco medicamentos, el mayor consumo se registró para benzodiacepinas (46\%), antihipertensivos (42\%), anti psicóticos (31\%) y anti inflamatorios no esteroideos (AINE) (18\%). En el $34 \%$ de los casos se confirmó la utilización inadecuada de los medicamentos y en el $38 \%$ una discordancia entre la indicación y la patología . En nuestro país se ha calculado que aproximadamente la cuarta parte del total del gasto en salud se destina a medicamentos ${ }^{9}$

Los factores de riesgo asociados a una RAM son el número de diagnósticos (multimorbilidad), el número de fármacos recibidos, el uso de drogas no apropiadas para la tercera edad

\section{y la falta de monitoreo de los tratamientos ${ }^{11,12}$.}

Se considera que la prescripción de un medicamento es inapropiada cuando ${ }^{13}$.

1) El riesgo de sufrir efectos adversos es superior al beneficio clínico, especialmente cuando hay evidencia de la existencia de alternativas terapéuticas más seguras y/o eficaces.

2) El medicamente se utiliza con una frecuencia o duración mayor al tiempo recomendado.

3) Existe un riesgo incrementado de interacción nociva con otros medicamentos, enfermedades o condiciones clínicas.

4) Existe duplicación de principios activos dentro de la misma clase farmacológica.

5) Se omiten prescripciones de fármacos beneficiosos para tratar o prevenir un problema de salud.

\section{Herramientas}

Dado el impacto de la prescripción inadecuada en el adulto mayor, a lo largo del tiempo se han propuesto diferentes estrategias para optimizar el uso de medicamentos. Estas herramientas se pueden dividir en implícitas y explicitas ${ }^{6}$ (ver tabla 1).

Los métodos implícitos se basan en el juicio clínico, evaluando cada medicamento considerando las características del paciente y la indicación de la prescripción. Resultan fiables, aunque laboriosos, y sus resultados dependen en gran medida del profesional que los aplica.

Los métodos explícitos utilizan criterios predefinidos basados en datos científicos y consenso de expertos para definir medicamentos potencialmente inapropiados. Constituye una herramienta más sencilla de utilizar y reproducible, permitiendo sistematizar la detección de prescripciones inapropiadas, aunque tienen la desventaja de requerir actualizaciones constantes.

Los primeros criterios explícitos, publicados en 1991, fueron los criterios de Beers que se han actualizado en varias ocasiones (1997, 2003 y 2012) y han sido en su última edición, asumidos como oficiales por la American Geriatric Society ${ }^{14}$. A pesar de su gran difusión internacional su aplicación en el ámbito Europeo ha sido limitada por varias razones, entre ellas, por no considerar la existencia de ciertas interacciones entre fármacos, la duplicidad terapéutica y la omisión de fármacos con indicación de uso. Por ello surgió en Europa la necesidad de desarrollar criterios más acorde con las necesidades y el arsenal terapéutico de ese continente. 
Tabla 1. Herramientas disponibles para la optimización de la prescripción de medicamentos.

\begin{tabular}{l|l}
\multicolumn{1}{c|}{ Implícitas } & \multicolumn{1}{c}{ Explícitas } \\
\hline $\begin{array}{l}\text { Improve Prescribing in the Elderly Tool (IPET; Canadá, 1997): describe los 14 errores } \\
\text { más frecuentes identificados por un panel de expertos en pacientes hospitalizados } \\
\text { mayores de } \mathbf{7 0} \text { años. }\end{array}$ & $\begin{array}{l}\text { Criterios Beers (1991): describe un listado de medicamentos apropiados para ser utilizados } \\
\text { con seguridad en las personas mayores. }\end{array}$ \\
\hline $\begin{array}{l}\text { Medication Appropriateness Index (MAl; Canadá, 1992 y 1994): describe diez criterios } \\
\text { relacionados con costos, dosis incorrecta y duración del tratamiento. }\end{array}$ & $\begin{array}{l}\text { Sreening Tool of Older Persons Prescriptions - Screning Tool to Alert doctors to Right i.e } \\
\text { appropriate indicated Treatment \& (STOPP- START; Irlanda, 2008): describen 65 y 22 } \\
\text { criterios. respectivamente. }\end{array}$ \\
\hline Assessing Care of Vulnerable Elders (ACOVE; EE.UU.) & Criterios de uso inapropiado en Australia (2008) \\
\hline Medication Screening Questionnaire (MSQ; EE.UU., 2011) & Medicación Potencialmente inadecuada en ancianos (Francia; 2007) \\
\hline & Norwegian General Practice (NORGEP; Noruega, 2009) \\
\hline
\end{tabular}

\section{Criterios STOPP-START}

Los criterios STOPP-START fueron publicados por primera vez en el año 2008 por Gallagher y Colaboradores, en Irlanda para su uso en diferentes ámbitos de atención, incluyendo los cuidados domiciliarios. Fueron pronto avalados por la Sociedad Europea de Geriatría publicando una versión en español en el año 2009 lo que ha colaborado en forma significativa a su difusión en los países de habla hispana ${ }^{13}$.

Estos criterios describen los errores más comunes de tratamiento y de omisión en la prescripción en adultos mayores, son fáciles de relacionar con el diagnóstico (ya que están agrupados por sistemas fisiológicos) y pueden integrarse en los sistemas informáticos de prescripción. Su aporte más novedoso con respecto a los criterios de Beers fue la inclusión de una lista que busca detectar la falta de uso de medicamentos potencialmente indicados (START).

La prevalencia de prescripción potencialmente inadecuada (PPI) según estos criterios, varía de acuerdo a la farmacopea de cada área y al ámbito asistencial donde se aplique. Esta prevalencia ( 15 al $70 \%$ ) es mayor en residencias que en hospitales y en la comunidad.

Según datos europeos, en medicina domiciliaria la mayor prevalencia de PPI según los criterios STOPP están relacionadas con el sistema nervioso central (benzodiacepinas y neurolépticos), sobre todo en pacientes con antecedentes de caídas; con el consumo excesivo de inhibidores de bomba de protones (IBP); con el uso de ácido acetil salicílico (AAS) en pacientes sin enfermedad cardiovascular; y con la duplicación terapéutica. Con respecto a la omisión en la prescripción (START), los medicamentos más comúnmente implicados fueron el calcio y la vitamina $D$ en pacientes con osteoporosis; estatinas y AAS en pacientes con diabetes mellitus y enfermedad cardiovascular, e inhibidores de la enzima convertidora de angiotensina (IECA) en insuficiencia cardiaca congestiva. Por su parte, en la comunidad, la mayor prevalencia de PPI según los criterios STOPP están relacionados con el uso prolongado benzodiacepinas de vida media larga, AAS sin indicación, AINE y fármacos del sistema cardiovascular. En relación a los criterios START, los antiagregantes y estatinas en diabetes mellitus (coexistiendo con uno o más factores de riesgo) o en pacientes con enfermedad cardiovascular, calcio y vitamina $D$ en osteoporosis y metformina en diabetes mellitus. En nuestro país se realizó un estudio en la ciudad de La Plata en el año 2010 que incluyó 141 pacientes de la comunidad mayores a 65 años donde se detectaron PPI, según criterios Beers, en un $25,5 \%$ de los casos. Las más frecuentes fueron el uso de benzodiacepinas (sobre todo clonazepam), seguido del uso de amiodarona. Según los criterios STOPP se detectaron $\mathrm{PPI}$ en el $24 \%$ de los casos. Los más frecuentes fueron el uso de IBP por más de ocho semanas, bloqueantes cálcicos en pacientes con constipación, AAS en dosis mayores a 150 $\mathrm{mg} / \mathrm{día}$, AINE por más de tres meses en artrosis, y benzodiacepinas en pacientes con caídas ${ }^{15}$.

La aplicación de los criterios STOPP/START en la revisión del tratamiento farmacológico en adultos mayores ha sido superior a los criterios de Beers 2003 para detectar o prevenir RAM ${ }^{16}$, aun cuando la nueva edición de los criterios de Beers pudo haber mejorado su rendimiento.

Utilizando los criterios STOPP, Cahir y col., encontraron que los pacientes con dos o más PPI tuvieron dos veces más riesgo de sufrir RAM y acudir a servicios de urgencias, presentando además una disminución de la calidad de vida relacionada con la salud ${ }^{17}$.

Otro estudio mostró que la aplicación de los criterios STOPP/START dentro de las 72 horas de la admisión hospitalaria de adultos mayores por enfermedades agudas no seleccionadas reduce en forma significativa las RAM, con una reducción del riesgo absoluto (RRA) del 9,3\% y un número necesario a tratar (NNT) de $11^{18}$. Un estudio multicéntrico realizado en residencias geriátricas mostró que la implementación de un programa educativo basado en el uso de criterios STOPP/START redujo el uso de antipsicóticos, el riesgo de delirium y caídas, logrando a su vez un menor uso de recursos sanitarios, incluidas las hospitalizaciones ${ }^{19}$.

En la actualidad se encuentra en marcha en Europa un ambicioso proyecto multicéntrico denominado SENATOR que intenta desarrollar y evaluar un potente software capaz de monitorizar el estado clínico y los tratamientos farmacológicos y no farmacológicos administrados a adultos mayores hospitalizados para reducir las PPI y las $\mathrm{RAM}^{20}$.

En el año 2014 se realizó la actualización de los criterios STOPP/START, la cual conto con la participación de 19 expertos en geriatría y farmacología geriátrica de 13 países de Europa (la versión 2008 incluyó solo expertos de Irlanda y Reino Unido). En este nuevo trabajo se volvió a usar el método de Delphi para la validación de los nuevos criterios. Se excluyeron 15 criterios de la primera versión (2008), que no contaban con evidencia consistente, se modificaron la mayoría de los criterios vigentes, y se agregaron 12 criterios STOPP (total 87 ) y 12 START (total 34) $)^{21}$.

En relación a los criterios START, salvo que el paciente se encuentre en situación terminal y necesite un enfoque paliativo, debieran considerarse la incorporación de ciertos tratamientos cuando estos se hayan omitido sin una razón clínica clara. Se asume que el prescriptor observará todas las contraindicaciones específicas de estos fármacos antes de recomendarlos en adultos mayores. 
Tabla 2. Actualización de los criterios STOPP. Intervenciones que son potencialmente inadecuadas en caso de cumplirse el/los enunciados correspondientes.

\section{Sección A. Indicación de medicación}

1. Cualquier medicamento prescripto sin una indicación basada en evidencia clínica.

2. Cualquier medicamento prescripto con una duración superior a la indicada, cuando la duración del tratamiento este bien definida.

3. Cualquier prescripción concomitante de dos fármacos de la misma clase, como dos AINE; inhibidores selectivos de la recaptación de serotonina (ISRS), diuréticos de asa, inhibidores de la enzima convertidora de angiotensina (IECA), anticoagulantes (debe optimizarse la monoterapia dentro de esa clase de fármacos antes de considerar un nuevo fármaco).

\section{Sección B. Sistema cardiovascular}

1. Digoxina en pacientes con insuficiencia cardíaca congestiva (ICC) con función sistólica conservada.

2. Amiodarona como tratamiento antiarrítmico de primera elección en las taquiarritmias supraventriculares (mayor riesgo de toxicidad que controlando la frecuencia).

3. Diuréticos tiazídicos cuando existe hipopotasemia (kalemia menor a $3 \mathrm{mEq} / \mathrm{L}$ ), hiponatremia (natremia menor a $130 \mathrm{mEq} / \mathrm{L}$ ) 0 hipercalcemia 0 con antecedentes de gota.

4. Antihipetensivos de acción central (metildopa, clonidina) salvo intolerancia o falta de eficacia a otras clases de antihipertensivos.

5. IECA o los antagonistas de los receptores de la angiotensina II (ARA 2) en pacientes con hiperkalemia.

6. Antagonistas de la aldosterona (espironolactona, esplerenona) junto con otros fármacos que pueden aumentar los niveles de potasio (IECA, ARA 2 amiloride, triamtereno) sin monitoreo del potasio (debería monitorizarse cada sis meses)

7. Inhibidores de la 5-fosfodiesterasa (sildenafilo, tadalafilo, verdenafilo) en ICC grave con hipotensión o asociado a nitratos (riego de colapso cardiovascular).

8. Diuréticos de asa para edemas maleolares sin evidencia de ICC. síndrome nefrótico, insuficiencia renal.

9. Diuréticos de asa como tratamiento de primera línea para hipertensión arterial o cuando existe incontinencia urinaria (empeora la incontinencia).

10. Betabloqueantes en presencia de bradicardia (frecuencia cardíaca < 50 latidos por minuto) o bloqueo aurículo-ventricular de segundo o tercer grado

11. Betabloqueantes en combinación con verapamilo o diltiazem (riesgo de bloqueo cardíaco).

\section{Sección C. Antiagregantes/Anticoagulantes}

1. AAS a dosis superiores a $160 \mathrm{mg} /$ día (aumento del riesgo de sangrado sin mayor eficacia).

2. AAS en pacientes con antecedentes de enfermedad ulcerosa péptica sin IBP.

3. AAS, clopidogrel, dipiridamol, antagonistas de vitamina K, inhibidores de la trombina o inhibidores del factor Xa en presencia de un riesgo significativo de sangrado (ejemplo: hipertensión grave no controlada, diátesis hemorrágica, sangrado reciente espontáneo significativo).

4. AAS más clopidogrel para la prevención secundaria del ictus salvo que el paciente tenga un stent coronario, un síndrome coronario agudo o una estenosis carotidea grave y sintomática (no hay evidencia de beneficios del clopidogrel en monoterapia).

5. AAS combinada con antagonistas de la vitamina K, Inhibidor de la trombina o del factor Xa en pacientes con fibrilación auricular crónica (la AAS no aporta beneficios)

6. Antiagregantes combinados con antagonistas de la vitamina K, inhibidor directo de la trombina o del factor Xa en pacientes con enfermedad coronaria, cerebrovascular o arterial periférica estables (el tratamiento combinado no aporta beneficios).

7. Ticlopina en cualquier circunstancia (clopidogrel y prasugrel tienen eficacia similar y menos efectos adversos).

8. Antagonistas de la vitamina $\mathrm{K}$, inhibidor directo de la trombina o del factor Xa para un primer episodio de trombosis venosa profunda no complicado durante más de seis meses

9. Antagonistas de la vitamina K, inhibidor directo de la trombina o del factor Xa para una primera embolia de pulmón no complicada durante más de 12 meses.

10. AINE en combinación de antagonistas de la vitamina $\mathrm{K}$, inhibidor directo de la trombina 0 del factor $\mathrm{X}$ a (riesgo hemorragia digestiva grave).

\section{Sección D. Sistema nervioso central y psicotrópicos}

1. Antidepresivos tricíclicos (ATC) en pacientes con demencia, glaucoma, trastornos de la conducción cardiaca, prostatismo o con antecedentes de retención aguda urinaria.

2. ATC como tratamiento de primera línea en depresión (mayor riesgo de efectos adversos que con ISRS).

3. Neurolépticos con efecto anticolinérgico moderados a graves (clorpromazina, clozapina, flifenazina) en pacientes con antecedentes de prostatismo o retención aguda de orina (alto riesgo de retención urinaria).

4. Benzodiacepinas durante más de cuatro semanas (no hay indicación para tratamientos más prolongados); riesgo de sedación prolongada, confusión, caídas, accidentes de tránsito (si el tratamiento

supera las cuatro semanas suspender en forma gradual para evitar el síndrome de abstinencia).

5. Antipsicóticos (distintos de quetiapina o clozapina) en pacientes con parkinsonismo o demencia por Cuerpos de Lewy (riesgo de efectos extrapiramidales)

6. Anticolinergicos/antimuscarinicos para tratar los efectos extrapiramidales de los neurolépticos.

7. Neurolépticos en pacientes dementes con trastornos de la conducta, salvo que sean graves y no respondan a otros tratamientos no farmacológicos (aumento del riesgo de ictus)

8. Anticolinérgicos en pacientes con delirium o demencia (empeoramiento de la cognición).

9. Neurolépticos como hipnóticos salvo que el trastorno del sueño se deba a demencia o psicosis (riesgo de confusión, caídas, hipotensión, efectos extrapiramidales).

10. Inhibidores de la acetilcolicolinesterasa en pacientes con antecedentes de bradicardia persistente (frecuencia cardíaca menor a 60 latidos por minuto), bloqueo cardiaco o sincopes recurrentes de etiología no clara, o que reciben tratamientos simultaneos con fármacos que bajan la frecuencia cardíaca como betabloqueantes, digoxina, diltiazem, verapamilo (trastornos de la conducción cardiaca sincope o lesiones)

11. Fenotiazidas como tratamiento de primera línea ya que existen alternativas más seguras y eficaces (son sedantes y tienen toxicidad antimuscarínica relevante en los mayores). Constituyen como excepción a este enunciado la proclorperazina en el tratamiento de náuseas, vómitos, vértigo; la clorpromazina para hipo persistente y levomepromazina como antiemético en cuidados paliativos).

12. Levodopa o agonistas dopaminérgicos para el temblor esencial (no hay evidencia de eficacia).

13. Antihistamínicos de primera generación (existen otros más seguros y menos tóxicos)

14. ISRS en pacientes con hiponatremia concurrente 0 reciente (natremia $<130 \mathrm{ma} / \mathrm{dl}$ ). 


\section{Sección E. Sistema Renal}

Los siguientes medicamentos son potencialmente inapropiados en el adulto mayor con enfermedad renal aguda o crónica por debajo de determinado nivel de filtrado glomerular (consultar vademécum locales y fichas técnicas).

1. Digoxina en dosis mayores a $125 \mathrm{ug} /$ día con tasa de filtrado glomerular (TGF) $<30 \mathrm{ml} / \mathrm{min}$ (riesgo de intoxicación digitálica si no se realiza monitoreo).

2. Inhibidores directos de la trombina (ej. dabigatran) con TFG <30 $\mathrm{ml} / \mathrm{min}$ (riesgo de sangrado).

3. Inhibidores del factor Xa (ej. rivaroxaban) con TFG $<15 \mathrm{ml} / \mathrm{m}$ (riesgo de sangrado).

4. AINE con TFG $<50 \mathrm{ml} / \mathrm{m}$ (riesgo de deterioro de la función renal.

5. Colchicina con $\mathrm{TFG}<10 \mathrm{ml} / \mathrm{m}$ (riesgo de toxicidad).

6. Metformina con TFG $<30 \mathrm{ml} / \mathrm{m}$ (riesgo de acidosis láctica).

\section{Sección F. Sistema gastrointestinal}

1. Hierro oral a dosis elementales superiores a $200 \mathrm{mg} /$ día (ej. hierro fumarato y sulfato $>600 \mathrm{mg} / d i ́ a$, hierro gluconato $>1.800 \mathrm{mg} /$ día). No hay evidencia de mayor absorción por encima de estas dosis).

2. Medicamentos que suelen causar estreñimiento (ej. fármacos anticolinérgicos, hierro oral, opioides, verapamilo, antiácidos con aluminio) en pacientes con estreñimiento crónico cuando existen otras alternativas que no estriñen.

3. IBP para la enfermedad ulcerosa péptica o esofagitis péptica erosiva a dosis terapéuticas plenas durante más de ocho semanas.

4. Metoclopramida en pacientes con parkinsonismo (empeora el parkinsonismo).

\section{Sección G. Sistema respiratorio}

1. Broncodilatadores antimuscarínicos (ej. ipratropio, tiotropio) en pacientes con antecedentes de glaucoma de ángulo estrecho (pueden exacerbar el glaucoma) u obstrucción del tracto urinario inferior (puede causar retención urinaria).

2. Betabloqueantes no cardioselectivos (orales o tópicos para el glaucoma) en pacientes con antecedentes de asma que requiere tratamiento (aumenta el riesgo de bronco espasmo).

3. Benzodiacepinas en pacientes con insuficiencia respiratoria aguda y crónica (riesgo de insuficiencia respiratoria).

4. Corticoides sistémicos en lugar de corticoides inhalados en la enfermedad pulmonar obstructiva crónica (EPOC) moderada a grave.

5. Teofilina como monoterapia para la enfermedad pulmonar obstructiva crónica (EPOC). (Existen alternativas más seguras).

\section{Sección H. Sistema musculo esquelética}

1. AINE (exceptuando los inhibidores selectivos de la COX-2) en pacientes con antecedentes de enfermedad ulcerosa péptica o hemorragia digestiva, salvo con uso simultaneo de antagonistas H2 0 IBP.

2. AlNE en pacientes con hipertensión grave o insuficiencia cardiaca (riesgo de exacerbación).

3. AINE a largo plazo (> tres meses) para el tratamiento sintomático de la artrosis cuando no se ha probado el paracetamol.

4. Inhibidores selectivos de la COX-2 en pacientes con enfermedad cardiovascular (aumento del riesgo de infarto e ictus).

5. AlNE con corticoides sin IBP a dosis profiláctica (aumenta el riesgo de enfermedad ulcerosa péptica).

6. Bifosfonatos orales en pacientes con enfermedades digestivas altas presentes o previas (ej. disfagia esofagitis, gastritis, duodenitis, enfermedad ulcerosa péptica o hemorragia digestiva alta, debido al riesgo de reaparición o de exacerbación de las mismas).

7. Corticoides a largo plazo (más de tres meses) como monoterapia en la artritis reumatoide.

8. AINE con corticoides sin IBP (aumenta el riesgo de enfermedad ulcerosa péptica).

9. Corticoides para el tratamiento de artrosis (salvo inyecciones intra-articulares periódicas en dolor monoarticular).

\section{Sección I. Sistema urogenital}

1. Fármacos antimuscarínicos vesicales en pacientes con demencia (aumenta el riesgo de confusión, agitación) o glaucoma de ángulo estrecho (exacerbación del glaucoma) o prostatismo crónico (riesgo de retención urinaria).

2. Bloqueantes alfa-1-adrenergico selectivos en pacientes con hipotensión ortostática sintomática o síncope miccional (riesgo de recurrencia del sincope).

\section{Sección J. Sistema endócrino}

1. Sulfonilureas de larga acción (ej:: glibenclamida, clorpropamida, glimeperida) en pacientes con diabetes mellitus tipo 2 (riesgo de hipoglucemia prolongada).

2. Tiazolidinedionas (ej. rosglitazona, pioglitazona) en pacientes con ICC (riesgo de exacerbación de ICC).

3. Andrógenos en ausencia de hipogonadismo primario o secundario (riesgo de toxicidad por andrógenos; no han demostrado beneficio fuera de la indicación de hipogonadismo).

4. Estrógenos con antecedentes de cáncer de mama o trombosis venosa (riesgo de recurrencia).

5. Estrógenos orales sin progestágenos en mujeres con útero intacto (riesgo de cáncer de útero).

6. Betabloqueantes en pacientes con diabetes mellitus con episodios frecuentes de hipoglucemia (enmascara los episodios).

\section{Sección K. Fármacos que aumentan en forma predecible el riesgo de caídas en personas mayores}

1. Benzodiacepinas (sedantes, pueden reducir el nivel de conciencia, deterioran el equilibrio).

2. Neurolépticos (pueden producir dispraxia en la marcha, parkinsonismo).

3. Vasodilatadores (bloqueantes alfa 1 adrenérgicos, antagonistas del calcio, nitratos de acción prolongada, IECA, ARA-II) en pacientes con hipotensión postural persistente (descenso recurrente superior a $20 \mathrm{mmHg}$ de presión sistólica, riesgo de sincope y caídas).

4. Hipnóticos-Z (ej. zoplicona, zolpidem, zaleplon; pueden causar sedación diurna prolongada, ataxia).

\section{Sección I. Analgésicos}

1. Uso de opiáceos potentes, orales o transdérmicos (morfina, oxicodona, fentanilo, buprenorfina, metadona, tramadol) como tratamiento de primera línea en el dolor leve (inobservancia de la escala de la OMS).

2. Uso de opioides pautados (no a demanda) sin asociar laxantes (riesgo de constipación grave).

3. Opiáceos de acción prolongada sin opioides de acción rápida para el dolor irruptivo (riesgo de persistencia del dolor).

\section{Sección N. Carga antimuscarínico/anticolinérgica}

1. Uso de dos o más fármacos antimuscarínicos/anticolinérgicos (ej. antiespasmódicos vesicales o intestinales, antihistamínicos de primera generación; debido al riesgo de toxicidad anticolinérgica).

Adaptado de: Delgado Silveira E. y Col. Rev Esp Geriatr Gerontol. 2015 Mar-Apr;50(2):89-96. 
Tabla 3. Actualización de los criterios START. Intervenciones que deben ser iniciadas en caso de cumplirse el/los enunciados correspondientes.

\section{Sección A. Sistema cardiovascular}

1. Antagonistas de la vitamina $K$, Inhibidor de la trombina 0 del factor Xa en presencia de fibrilación auricular crónica.

2. AAS en presencia de una fibrilación auricular crónica, cuando los antagonistas de la vitamina $K$, inhibidor de la trombina o del factor Xa estén contraindicados.

3. Antiagregantes (AAS, clopidogrel, prasugrel o ticagrelor) en pacientes con antecedentes bien documentados de enfermedad coronaria, cerebral 0 arterial periférica.

4. Tratamiento antihipertensivos cuando la presión arterial sistólica sea habitualmente superior a $160 \mathrm{mmHg}$ y/o la presión diastólica sea habitualmente > $90 \mathrm{mmHg}$ (>140 mmHg y $90 \mathrm{mmHg}$ si tiene diabetes mellitus)

5. Estatinas en pacientes con antecedentes bien documentados de enfermedad arteriosclerótica coronaria, cerebral o arterial periférica, salvo que el paciente esté en situación de final de vida o su edad sea mayor de 85 años.

6. IECA en la insuficiencia cardiaca sistólica y/o cardiopatía isquémica bien documentada.

7. Bloqueadores beta en la cardiopatía isquémica.

8. Bloqueadores betas apropiados (bisoprolol, nebivolol, metoprolol o carvedilol) en ICC sistólica estable.

\section{Sección B. Sistema respiratorio}

1. Corticosteroides inhalados pautados en el asma o EPOC moderado a grave, cuando el volumen de espiración forzada (FEV1) es inferior al $50 \%$ y existen frecuentes exacerbaciones que requieren corticoides orales.

2. Oxigenoterapia domiciliaria continua en la hipoxemia crónica (p02 <8,0 kPa [60mmHg] 0 saturación de $02<89 \%)$.

3. Agonista B2 0 antimuscarínicos inhalatorios pautados (ej. ipratropio) en el asma o EPOC leve a moderado.

\section{Sección C. Sistema nervioso central y ojos}

1. Levodopa o un agonista dopaminergico en la enfermedad de Parkinson idiopática con deterioro funcional y discapacidad secundaria.

2. Antidepresivos no tricíclicos en presencia de síntomas depresivos mayores persistentes.

3. Inhibidores de la acetilcolinesterasa (donepecilo, rivastigmina, galantamina) para la enfermedad de Alzheimer leve-moderado o la demencia de por cuerpos de lewy (rivastigmina).

4. Prostaglandinas, prostamina o betabloqueantes tópicos para el glaucoma primario de ángulo abierto.

5. ISRS (inhibidores selectivos de la recaptación de noradrenalina o pregabalina si los ISRS están contraindicados) para la ansiedad grave persistente que interfiere con la independencia funcional.

6. Antagonistas dopaminérgicos (pramipexol) para síndrome de piernas inquietas una vez descartado como causas la deficiencia de hierro y la enfermedad renal grave.

\section{Sección D. Sistema gastrointestinal}

1. Suplementos de fibra (ej. salvado, metilcelulosa) en la diverticulosis sintomática crónica con antecedentes de estreñimiento.

2. IBP en la enfermedad por reflujo gastroesofágico grave o estenosis péptica que requiera dilatación.

\section{Sección E. Sistema musculo esquelético}

1. Fármacos antirreumáticos modificadores de la enfermedad en la artritis reumatoide activa incapacitante.

2. Suplementos de calcio y vitamina D en pacientes con osteoporosis conocida y/o fracturas por fragilidad previas y/o densidad mineral ósea con T score inferiores a -2,5 en múltiples puntos.

3. Antirresortivos 0 anabolizantes óseos (ej. bifosfonatos, ranelato de estroncio, teriparatida, denosumab) en pacientes con osteoporosis conocida cuando no existan contraindicaciones para su uso.

4. Suplementos de vitamina D en pacientes mayores que no salen de la casa, sufren caídas o tienen osteopenia ( $T$ score -1 a -2.5 en múltiples puntos)

5. Inhibidores de la xantino oxidasa (ej. allopurinol) en pacientes con antecedentes de episodios de gota recurrentes.

6. Suplementos de ácido fólico en pacientes que toman metotrexato.

\section{Sección F. Sistema endocrinológico}

1. IECA o ARA-2 en pacientes con diabetes mellitus con evidencia de enfermedad renal (proteinuria en una tira reactiva o micro albuminuria $(>30 \mathrm{mg} / 24 \mathrm{~h})$ con 0 sin datos bioquímicos de enfermedad renal. Sección G. Sistema Genitourinario

1. Bloqueantes alfa 1 (tamsulosina) para el prostatismo sintomático cuando no se considera necesaria la prostatectomía.

2. Inhibidores de la 5 alfa reductasa para el prostatismo sintomático cuando no se considera necesaria la prostatectomía.

3. Estrógenos tópicos vaginales o pesario con estrógenos para la vaginitis atrófica sintomática.

\section{Sección H. Analgésicos}

1. Opioides potentes en el dolor moderado o severo cuando el paracetamol, los AINES o los opioides de baja potencia no son apropiados para la gravedad del dolor.

2. Laxantes en pacientes que reciben opioides de forma regular.

\section{Sección I. Vacunas}

1. Vacuna trivalente anual contra la gripe estacional.

2. Vacuna antineumocócica cada cinco años.

Adaptado de: Delgado Silveira E. y Col. Rev Esp Geriatr Gerontol. 2015 Mar-Apr,50(2):89-96. Si bien los nuevos criterios STOPP-START son más claros e incluyen un mayor número de fármacos hay otros puntos incluidos en otras herramientas que son también importantes a considerar en la prescripción en el adulto mayor, como son: el costo y la practicidad del tratamiento (MAI, MSQ), la elaboración de una lista de medicamentos, el monitoreo de algunos fármacos (ACOVE), el ajuste de la dosis según la función renal y hepática (MSQ), y la valoración de interacciones medicamentosas prevalentes (NORGEP).

\section{Conclusión}

Los criterios STOPP-START han demostrado ser una buena herramienta para la detección de prescripciones potencialmente inadecuadas y para la mejora de la calidad de prescripción en diferentes ámbitos de atención.

Como toda herramienta no reemplaza el juicio clínico pero sirve como una guía para la prescripción racional de medicamentos, en este acto es clave tener en cuenta además la expectativa de vida, el tiempo hasta el beneficio del fármaco, las metas de cuidado y las preferencias del paciente. 


\section{Referencias}

1. Baum C. y Col. Drug use in the United States in 1981. JAMA. 1984 Mar 9; 251(10):1293-7.

2. Gavilán-Moral E. y Col. Polypharmacy in frail elderly patients: is deprescribing the answer?. Rev Esp Geriatr Gerontol. Jul-Aug; 47(4):162-7.

3. Page RL. y Col. Inappropriate prescribing in the hospitalized elderly patient: defining the problem, evaluation tools, and possible solutions. Clin Interv Aging.5:75-87.

4. Wynne HA. y Col. Drug treatment in an ageing population: practical implications. Maturitas. 2010 Jul;66(3):246-50.

5. Borchelt M. Important aspects of geriatric pharmacotherapy. Bundesgesundheitsblatt Gesundheitsforschung Gesundheitsschutz. 2005 May; 48(5):593-8.

6. Spinewine A. y Col. appropriate prescribing in elderly people: how well can it be measured and optimised? Lancet. 2007 Jul 14; 370(9582):173-84.

7. Soumerai SB. y Col. Inadequate prescription-drug coverage for Medicare enrolees-a call to action. N Engl J Med. 1999 Mar 4; 340(9):722-8.

8. Lazarou J. y Col. Incidence of adverse drug reactions in hospitalized patients: a meta-analysis of prospective studies. JAMA. 1998 Apr 15;279(15):1200-5.

9. Ministerio de Economía y Ministerio de Salud de la Nación. Estimación del gasto en salud (documento en línea). 2001.

Disponible en URL: http://www.mecon.gov.ar/peconomica/basehome/gsalud.pdf

10. Gustavo H. y Col. Utilización de medicamentos en ancianos Institucionalizados en una ciudad de la Pcia de Bs As. Latin American Journal of Pharmacy. 2010;29(4):495-503.

11. Lindley CM. y Col. Inappropriate medication is a major cause of adverse drug reactions in elderly patients. Age Ageing. 1992 Jul; 21(4):294-300

12. Klarin I. y Col. The association of inappropriate drug use with hospitalisation and mortality: a population-based study of the very old. Drugs Aging. 2005;22(1):69-82.

13. Delgado Silveira E. y Col. Inappropriate prescription in older patients: the STOPP/START criteria. Rev Esp Geriatr Gerontol. 2009 Sep-Oct; 44(5):273-9.

14. Fick D, y Col. American Geriatrics Society updated Beers Criteria for potentially inappropriate medication use in older adults. J Am Geriatr Soc. 2012 Apr; 60 (4):616-31

15. Regueiro M. y col. Use of medication in elderly people don't institutionalized. Rev Peru Med Exp Salud Pública. 2011 Dec;28(4):643-7.

16. Delgado Silveira E. y Col. Improving drug prescribing in the elderly: a new edition of STOPP/START criteria. Rev Esp Geriatr Gerontol. 2015 Mar-Apr;50(2):89-96.

17. Cahir C. y Col. Potentially inappropriate prescribing and adverse health outcomes in community dwelling older patients. Br J Clin Pharmacol. 2014 Jan;77(1):201-10.

18. Denis O'Mahony UCC. Prevention of Adverse Drug Events (ADEs) in Hospitalised Older Patients. 2013.

19. Garcia-Gollarte F. y Col. An educational intervention on drug use in nursing homes improves health outcomes resource utilization and reduces inappropriate drug prescription. J Am Med Dir Assoc. 2014 Dec;15(12):885-91.

20. Development and clinical trials of a new Software ENgine for the Assessment \& optimization of drug and non-drug Therapy in Older peRsons. SENATOR. Disponible en URL: http://www.senatorproject.eu/ (último acceso, diciembre 2015).

21. O' Mahony D. y Col. STOPP/START criteria for potentially inappropriate prescribing in older people: version 2. Age Ageing. 2015 Mar;44(2):213-8.

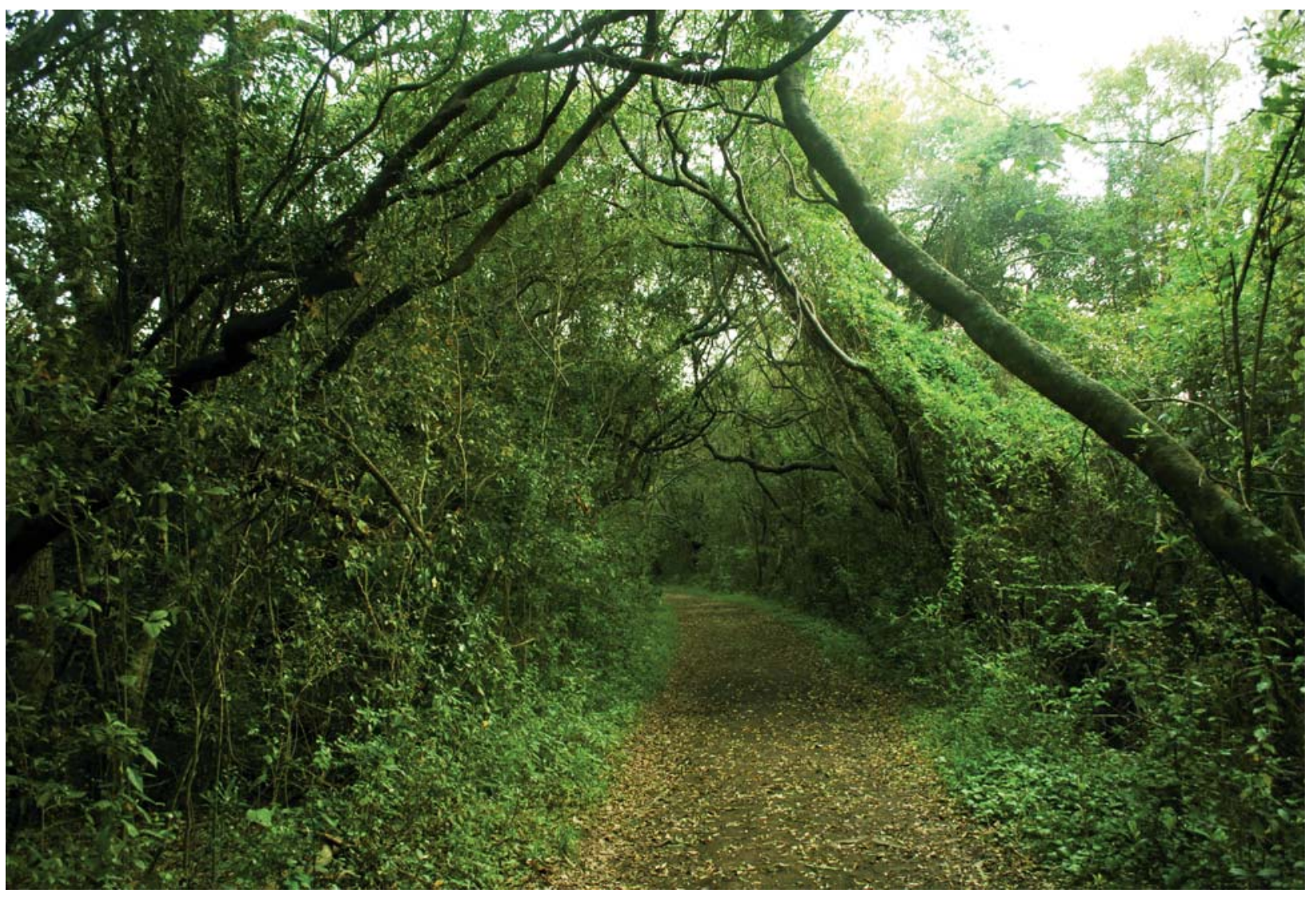

Fotografía: Silvia Spina 